\title{
TOPOLOGICAL PROPERTIES OF THE BLOCK NUMERICAL RANGE OF OPERATOR MATRICES
}

\author{
Agnes Radl And Manfred P. H. WolfF
}

\begin{abstract}
We show that the block numerical range of an $n \times n$-operator matrix $\mathscr{A}$ corresponding to an operator $A$ on the Banach space $X$ with respect to a decomposition $X=\Pi X_{j}$ has at most $n$ connected components. We then characterize operator matrices with finite block numerical range. As an important tool we prove an inclusion theorem for the block numerical ranges of the principal submatrices of $\mathscr{A}$.
\end{abstract}

Mathematics subject classification (2010): 47A10, 47A12, 47A75, 47B65, 15A60, 46B08, 46B42. Keywords and phrases: Block numerical range, numerical range, spectrum, resolvent.

\section{REFERENCES}

[1] F. F. Bonsall, J. Duncan, Numerical Ranges of Operators on Normed Spaces and of Elements of Normed Algebras, London Mathematical Society Lecture Note Series 2, Cambridge University Press, London, 1971.

[2] R. A. Brualdi, H. J. Ryser, Combinatorial Matrix Theory, Cambridge University Press, Cambridge, 1991.

[3] J. Diestel, Geometry of Banach Spaces - Selected Topics, Lecture Notes in Mathematics 485, BerlinHeidelberg-New York, 715 Springer, 1975.

[4] E. Freitag, R. Bus am, Complex Analysis, Springer Verlag Berlin-Heidelberg, 2005.

[5] P. KALLUS, The block numerical range of operator functions in Banach spaces, TU Berlin, Bachelor's thesis, Supervisor: K.-H. Förster, 2011.

[6] J. Lindenstrauss, L. Tzafriri, Classical Banach spaces, II. Function spaces, Ergebnisse der Mathematik und ihrer Grenzgebiete [Results in Mathematics and Related Areas], 97, Berlin-New York: Springer-Verlag, 1979.

[7] A. RADL, M. P. H. WOLFF, On the block numerical range of operators on arbitrary Banach spaces, (2016), Oper. Matrices 12 (2018), 229-252.

[8] A. Salemi, H. R. AfShin, Quadratic numerical range of block operator matrices, Far East Journal of Mathematical Sciences (FJMS) 10 (2003), 99-103.

[9] C. Tretter, M. Wagenhofer, The block numerical range of an $n \times n$ block operator matrix, SIAM J. Matrix Anal. Appl. 24 (2003), 1003-1017.

[10] C. TRETTER, Spectral Theory of Block Operator Matrices and Applications, Imperial College Press, London, 2008.

[11] M. Wagenhofer, Block Numerical Ranges, Dissertation, Universität Bremen, 2007. 